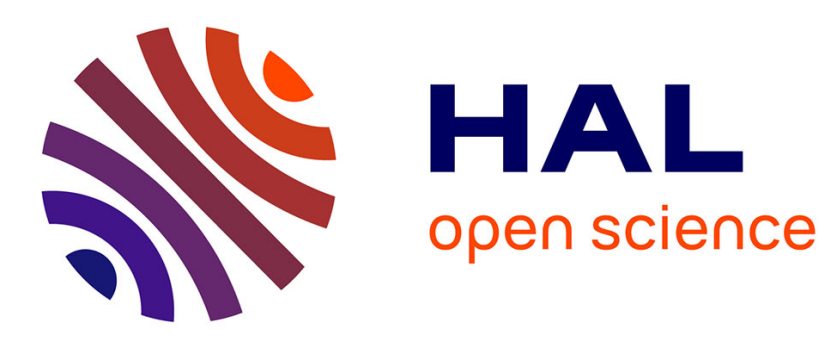

\title{
Synthesis Method for Matching Filters
}

David Martínez Martínez, Fabien Seyfert, Martine Olivi, Stéphane Bila, François Torres, Johann Sence

\section{To cite this version:}

David Martínez Martínez, Fabien Seyfert, Martine Olivi, Stéphane Bila, François Torres, et al.. Synthesis Method for Matching Filters. 2017 IEEE MTT-S International Microwave Symposium (IMS), Jun 2017, Honololu, United States. pp.1930-1933, 10.1109/MWSYM.2017.8059040 . hal-01420636

\section{HAL Id: hal-01420636 https://hal.science/hal-01420636}

Submitted on 6 Mar 2017

HAL is a multi-disciplinary open access archive for the deposit and dissemination of scientific research documents, whether they are published or not. The documents may come from teaching and research institutions in France or abroad, or from public or private research centers.
L'archive ouverte pluridisciplinaire HAL, est destinée au dépôt et à la diffusion de documents scientifiques de niveau recherche, publiés ou non, émanant des établissements d'enseignement et de recherche français ou étrangers, des laboratoires publics ou privés. 


\section{Synthesis Method for Matching Filters}

\author{
David Martínez Martínez, Fabien Seyfert \\ and Martine Olivi \\ INRIA Sophia-Antipolis Méditerranée \\ 06902 Sophia Antipolis, FRANCE
}

\author{
Stéphane Bila, François Torres \\ and Johann Sence \\ XLIM UMR7252 Université de Limoges / CNRS \\ 87060 Limoges, FRANCE
}

\begin{abstract}
The aim of this paper is to develop an exact synthesis technique for matching filters in connexion with the use of single band antennas. A certified algorithm based on Youla's matching theory and convex optimization is presented. A practical example is considered with the synthesis of a matching filter in SIW technology used behind a microstrip patch antenna at $1.5 \mathrm{Ghz}$.

Index Terms-Filter, Antenna, Synthesis, Matching, SIW, Patch Antenna
\end{abstract}

\section{INTRODUCTION}

The synthesis of filters and antennas present in microwave reception or emission chains are usually done by assuming a fix resistive reference (usually $50 \mathrm{Ohm}$ ) at their connecting ports. The antenna's mismatch is then either ignored or compensated by an additional matching network, resulting either in a degradation of the energy efficiency or in an increased footprint of the overall hardware. The aim of this paper is to develop a synthesis technique for matching filters, allowing to handle the matching and filtering requirements in a single filter and rendering superfluous the use of an extra matching network.

The literature about matching problems is vast. In the fifties and sixties Bode, Fano and Youla [1], [2], [3] pioneered their broad-band matching theory based on the use of the Darlington two port equivalent and extraction procedures. Despite its undeniable elegance this approach did not result in massive practical applications, mainly because of its complexity and the relative rigidity of its induced practical implementations. The theory was for example used to synthesize matching networks with a Tchebychev type transducer power gain [4], nevertheless this type of responses is known to be non optimal in terms of matching performances unless the load is a constant impedance. This approach was therefore progressively replaced by the optimization based "real frequency" technique of Carlin [5] which is more oriented to practical applications. These methods however focuses only on matching requirement of the designed network and do not allow to impose rejection specifications in specified frequency bands. Moreover, like any non-convex optimization approach, this method does not guarantee the optimality of the obtained transducer gain function.

The presented approach proposes a guaranteed optimal synthesis technique yielding the best possible matching filter for a given load, provided its reflection coefficient can be reasonably approached by a rational function of degree one in the band of interest. Specifications are, like in classical filter synthesis, given in terms of return loss and rejection levels. We first detail the algorithmic structure of our synthesis procedure based on Youla's matching machinery coupled with convex optimization techniques. The method is then demonstrated at hand of a single band planar patch antenna and the design of a matching filter in SIW technology.

\section{DERIVING NECESSARY AND SUFFICIENT CONDITION FOR THE LOAD EXTRACTION}

Following Youla's and Fano's approach, our method deals with the synthesis of the overall system of degree $N+1$ composed of a matching filter of degree $N$ chained at port 2 with a two-ports loss-less reciprocal load (Fig. 1) of degree one. The global system is represented by the scattering matrix $S^{C}$ meanwhile the matching filter and the load are represented by the matrices $S^{F}$ and $S^{A}$ respectively. If the passive load is known by its reflection coefficient $S_{11}^{A}$ at port 1, the Darlington equivalent can be constructed yielding a loss-less two port with same reflection coefficient.

Necessary and sufficient conditions in order to allow for the extraction of a specified load at port 2 of $S^{C}$, are readily given by deriving the expression of the reflection coefficient $S_{22}^{C}$ :

$$
S_{22}^{C}=S_{22}^{A}+\frac{S_{21}^{A} S_{12}^{A} S_{22}^{F}}{1-S_{11}^{A} S_{22}^{F}}
$$

At the transmission zero ${ }^{1} \omega_{0}$ of the load $\left(S_{12}^{A}\left(\omega_{0}\right)=\right.$ $\left.S_{21}^{A}\left(\omega_{0}\right)=0\right)$, (1) yields following conditions on $S^{C}$ :

$$
\begin{aligned}
S_{22}^{C}\left(\omega_{0}\right) & =K_{0} & \left|K_{0}\right| & =1 \\
-\Im\left[\frac{d}{d \omega} \log S_{22}^{C}(\omega)\right] & \leq K_{1} & K_{1} & \geq 0
\end{aligned}
$$

where $\Im$ represents the imaginary part and $K_{0}$ and $K_{1}$ are defined as follows:

$$
\begin{aligned}
& K_{0}=S_{22}^{A}\left(\omega_{0}\right) \\
& K_{1}=-\Im\left[\frac{d}{d \omega} \log S_{22}^{A}(\omega)\right] \quad \omega=\omega_{0}
\end{aligned}
$$

The left hand term of (3) representing therefore the angular derivative of $S_{22}^{C}$ meanwhile $K_{1}$ stands for the angular derivative of $S_{22}^{C}$.

Moreover note $S_{22}^{C}$ can be multiplied by any uni-modular value without modifying (3). If the class of functions where $S_{22}^{C}$ is sought for allows for such a multiplication, (2) can be

\footnotetext{
${ }^{1}$ We suppose here that the TZ occurs at finite frequency. The same framework with slightly different formulas can we developed when the TZ occurs at infinity
} 


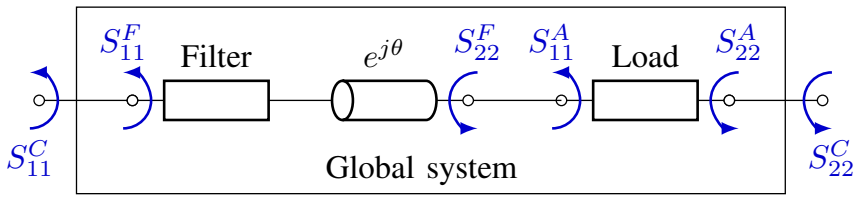

Fig. 1. Global system structure and scattering parameters.

replaced by the fact $\omega_{0}$ is also a transmission zero of $S^{C}$. Thus the necessary conditions over $S^{C}$ become:

$$
\begin{aligned}
S_{12}^{C}\left(\omega_{0}\right)=S_{21}^{C}\left(\omega_{0}\right) & =0 \\
-\Im\left[\frac{d}{d \omega} \log S_{22}^{C}(\omega)\right] & \leq K_{1} \quad K_{1} \geq 0
\end{aligned}
$$

A core result of Youla's matching theory is that these necessary conditions are indeed sufficient to ensure that the load $S^{A}$ can be extracted from the scattering matrix $S^{C}$ at port 2 [5].

\section{MATChing Filter SyNthesis PROBlem AS A CONVEX OPTIMIZATION PROBLEM}

\section{A. Deriving equations}

We suppose [6] that the loss-less two ports have rational frequency responses and can therefore be represented by means of the Belevitch form, that is:

$$
S^{C}=\frac{1}{q}\left(\begin{array}{cc}
\epsilon p^{*} & -\epsilon r^{*} \\
r & p
\end{array}\right)
$$

where $q=q(p)$ is the unique Hurwitz polynomials satisfying the spectral equation $q q^{*}=p p^{*}+r r^{*}, \epsilon$ is an uni-modular constant and $r=-\epsilon r^{*}$. The squared modulus of $S_{22}^{C}=\frac{p}{q}$ can be expressed as:

$$
\left|S_{22}^{C}\right|^{2}=\frac{p p^{*}}{p p^{*}+r r^{*}}=\frac{1}{1+\left(\frac{p p^{*}}{r r^{*}}\right)^{-1}} .
$$

Therefore, as used heavily in classical filter synthesis [6], uniform constraints on the modulus of $S_{22}^{C}$ can be casted to uniform constraints on the filtering function $p p^{*} / r r^{*}$. Note that condition (6) translates into the fact that $\omega_{0}$ is a root of $r$. As for the possible other finite transmissions zeros of $S^{C}$, we suppose as in classical filter synthesis, that they are fixed by the user and therefore not part of the optimization process. We set $S_{22}^{C}$ to be of maximal order $N+1$. Finding the reflection coefficient $S_{22}^{C}$ with the best return loss level on the frequency band $B_{1}$ with a specified rejection on the stop band $B_{2}$, while ensuring that the load with matrix $S^{A}$ can be extracted from $S^{C}$ (at port 2) can therefore be formulated as following optimization problem:

Problem 1. Find $L_{o p t}=\min _{p \in \mathbb{P}^{N+1}}\left(\max _{\omega \in B_{1}} \frac{p p^{*}}{r r^{*}}(\omega)\right)$, subject to:

$$
\begin{aligned}
\frac{p p^{*}}{r r^{*}}(\omega) & \geq \Gamma & \omega & \in B_{2} \\
-\Im\left[\frac{d}{d \omega} \log \frac{p}{q(p)}(\omega)\right] & \leq K_{1} & \omega & =\omega_{0}
\end{aligned}
$$

In the latter $\mathbb{P}^{N}$ is the set of polynomials of degree at most $\mathrm{N}$, meanwhile $\Gamma$ can be computed from the specified rejection constraint:

$$
\Gamma=\frac{1}{\gamma^{-1}-1}
$$

with $\gamma$ representing the rejection constraint on $\left|S_{22}^{C}\right|^{2}$.

This problem presents a remarkable property,

- There exist a minimum phase polynomial $p$ solution to the problem which does not vanish at $\omega=\omega_{0}$. Thus $p$ can be restricted to be a Hurwitz polynomial in the broad sense, that is with roots in the closed left half plane.

\section{B. A convex problem}

Problem 1 is not convex since it involves the computation of the spectral factorization of $q q^{*}$ needed in condition (11). Nevertheless due to the minimum phase property of $p,(11)$ can be expressed as a function of the modulus function $|p / q|^{2}$ by means of the Hilbert Transform. Eventually with the change of variable: $p p^{*}=P, r r^{*}=R$, condition (11) is rewritten as $f(P) \leq K_{1}$ where $f(P)$ is the function:

$$
f(P)=\frac{1}{2 \pi} \int_{\mathbb{R}} \log \left(1+\frac{R(\omega)}{P(\omega)}\right)\left(\omega-\omega_{0}\right)^{-2} d \omega .
$$

Again, condition (6) implies that $R(\omega)$ presents a double zero at $\omega_{0}$, which cancels the double singularity at $\omega=\omega_{0}$ rendering the integral definite if $P\left(\omega_{0}\right) \neq 0$.

Problem 1 restates as:

Problem 2. Find $L_{o p t}=\min _{P \in \mathbb{P}_{+}^{M}}\left(\max _{\omega \in B_{1}} \frac{P}{R}(\omega)\right)$, subject to:

$$
\begin{aligned}
P(\omega) & \geq \Gamma \cdot R(\omega) \quad \omega \in B_{2} \\
f(P) & \leq K_{1}
\end{aligned}
$$

where $\mathbb{P}_{+}^{M}$ denote the positive polynomials of degree at most $M$ with $M=2 N+2$.

This problem has the following properties:

1) The space $\mathbb{P}_{+}^{M}$ is a convex set.

2) $f(P)$ with $P \in \mathbb{P}_{+}^{M}$ is strictly convex as function of $P$. The convexity guarantees therefore the absence of local minima meanwhile uniqueness of the solution can be obtained from the strict convexity.

3) The optimum return loss level is reached in $N+2$ points.

As an illustrative example to show the interest of solving the previous problem, Figure 2 shows the optimum response in terms of matching satisfying the following specifications: an interval $B_{1}$ (passband) at $-1 \leq \omega \leq 1$, a required rejection of $-50 \mathrm{~dB}$ in the interval $B_{2}$ (stopband) composed of the union of intervals $-5 \leq \omega \leq-3$ and $3 \leq \omega \leq 5$ and an antenna providing a derivative $K_{1}=1$. An optimum return loss level of $-11.86 \mathrm{~dB}$ is attained. Conversely, if the global system is designed by means of a Tchebyshev response satisfying the same specifications, because of the constraint over the area, a return loss level of $-7.6 \mathrm{~dB}$ is obtained (Fig. 2). 

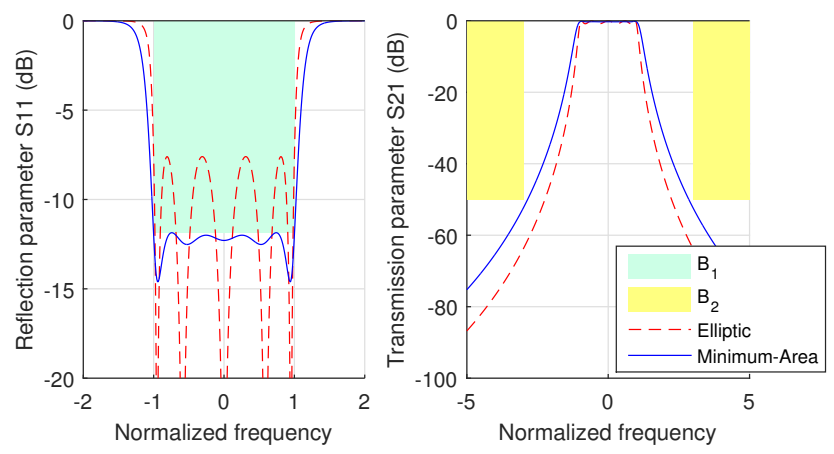

Fig. 2. Example of filter responses satisfying the constraints.

\section{A Practical Algorithm}

Eventually problem 2 can be solved efficiently by using its dual version which involves only linear constraints.

Problem 3. Find $K_{o p t}=\min _{P \in \mathbb{P}_{+}^{M}} f(P)$, subject to:

$$
\begin{array}{lll}
P(\omega) \leq L \cdot R(\omega) & L \geq 0 & \omega \in B_{1} \\
P(\omega) \geq \Gamma \cdot R(\omega) & \Gamma \geq 0 & \omega \in B_{2}
\end{array}
$$

Properties of this dual problem are listed below:

1) Problem 3 is equivalent to Problem 2 in the following sense: if $P_{o p t}$ solves Problem 2 with optimal criterion $L_{o p t}$ then it is also the solution of Problem 3 with $L=$ $L_{o p t}$ and optimal criterion $K_{o p t}=K_{1}$.

2) The parameter $L$ can be chosen in the interval $\left[L_{\text {min }}, \infty[\right.$. Here $L_{\min }$ is the value of $L_{o p t}$ obtained in Problem 2 when constrained (15) is removed, that is by means of a quasi-elliptic response that satisfies the rejection constraint (14). We note $K_{\max }$ the optimal value $K_{\text {opt }}$ obtained in Problem 3 with $L=L_{m i n}$.

3) For any positive value $\left.\left.K_{1} \in\right] 0, K_{\max }\right]$, the value of parameter $L$ in the dual problem can be adjusted by dichotomy to ensure that its optimal criterion $K_{\text {opt }}=K_{1}$. Therefore Problem 2 can be solved at hand of Problem 3.

The results presented in this paper have been computed with the fmincon function of MATLAB. Handling of the linear constraints (16) and (17) is made by the discretization of the frequency intervals $B_{1}$ and $B_{2}$ and the positivity of $P(\omega)$ is ensured by a set of additional constraints $P\left(\omega_{i}\right)>0$ where $\omega_{i}$ is a given number of control points properly distributed around the passband.

\section{Different kinds of solutions}

In Problem 2, two different kinds of solutions can be distinguished depending on which constraints are active:

- Quasi-elliptic response: if (15) is not active, the solution $P_{o p t}$ solves a classical filter synthesis problem. If $p_{o p t}$ is defined as $p_{\text {opt }} p_{\text {opt }}^{*}=P_{\text {opt }}$ then $p_{\text {opt }}$ is the Tchebychev polynomial of the interval $B_{1}$ with weight $1 /|r|$ [6].

- Minimum-area responses: if (15) is active, the solution realizes the best possible matching level under the specified selectivity requirement.

\section{RESUlTS}

To show the practical interest of the above algorithm we consider the case of a microstrip patch antenna for a GNSS receiver. The specifications are the coverage of the band $L_{1}$ (from $1.55 \mathrm{GHz}$ to $1.6 \mathrm{GHz}$ ) with a required rejection of $-20 d B$ at $f c_{1} \leq 1.495$ and $f c_{2} \geq 1.625$. At hand of these specifications our algorithm yields the best possible response $S^{C}$ of order 4 (Fig. 3). After deembedding the antenna at port 2 of the matrix $S^{C}$, the Belevitch model of the matching filter of order 3 is obtained. As a comparison a classical

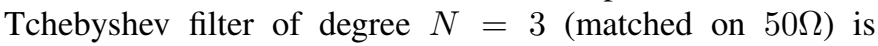
designed to satisfy the previous specifications with the lowest return loss. The reflection coefficient of the overall system made of this Tchebyshev filter plugged on the antenna is also plotted in dotted lines on Fig. 3 showing the improvement in the power transfer towards the antenna obtained by means of the matching filter at the border of transmission band. In both cases the transducer gain $(T G)$ of the antenna is considered to compute the transmission of the global system [5]:

$$
T G=1-\left|S_{11}\right|^{2}
$$

Note the system $S^{C}$ within the passband when using a Tchebyshev filter matched on $50 \Omega$ corresponds approximately to the response of the antenna referenced to $50 \Omega$ (Fig. 3).

The matching filter is synthesized as an in-line coupled resonator filter realized in $S I W$ planar technology fed with $C P W G$ input and output lines (Fig. 4) by using the substrate Rogers RT/duroid 6010LM. In addition, a transmission line of $10.5 \mathrm{~mm}$ has been required to adjust the phase of $S_{22}^{C}$.

The practical design of the filter is done via the classical coupling matrix approach [6] and using the full wave simulation software Ansoft Electronic Desktop. Note that the target coupling matrix $\left(M_{T}\right)$ is obtained from the previous algorithm.

$$
M_{T}=\left[\begin{array}{rrrrr}
0 & 1.195 & 0 & 0 & 0 \\
1.195 & 0 & 1.018 & 0 & 0 \\
0 & 1.018 & -0.007 & 0.7 & 0 \\
0 & 0 & 0.7 & -0.404 & 1.009 \\
0 & 0 & 0 & 1.009 & 0
\end{array}\right]
$$

However this kind of filtering functions differs from the classical Tchebyshev responses in the sense that they do not present all reflection zeros distributed on the frequency axis but inside the complex plane. For this reason and in order to achieve a good agreement between the circuital response and the EM response, the design has been assisted with the circuit extraction software PRESTO-HF [7] that compares the target coupling matrix with the one extracted from the EM response adjusting the physical dimensions in consequence. The final error in the coupling matrix is computed as $E=M_{T}-M_{E M}$ :

$$
E=\left[\begin{array}{rrrrr}
0 & -3.2 & 0 & 0.5 & 0 \\
-3.2 & -0.4 & 0.3 & -0.3 & 0.5 \\
0 & 0.3 & -1.1 & 0.8 & 0 \\
0.5 & -0.3 & 0.8 & -0.6 & -2.1 \\
0 & 0.5 & 0 & -2.1 & 0
\end{array}\right] \cdot 10^{-2}
$$



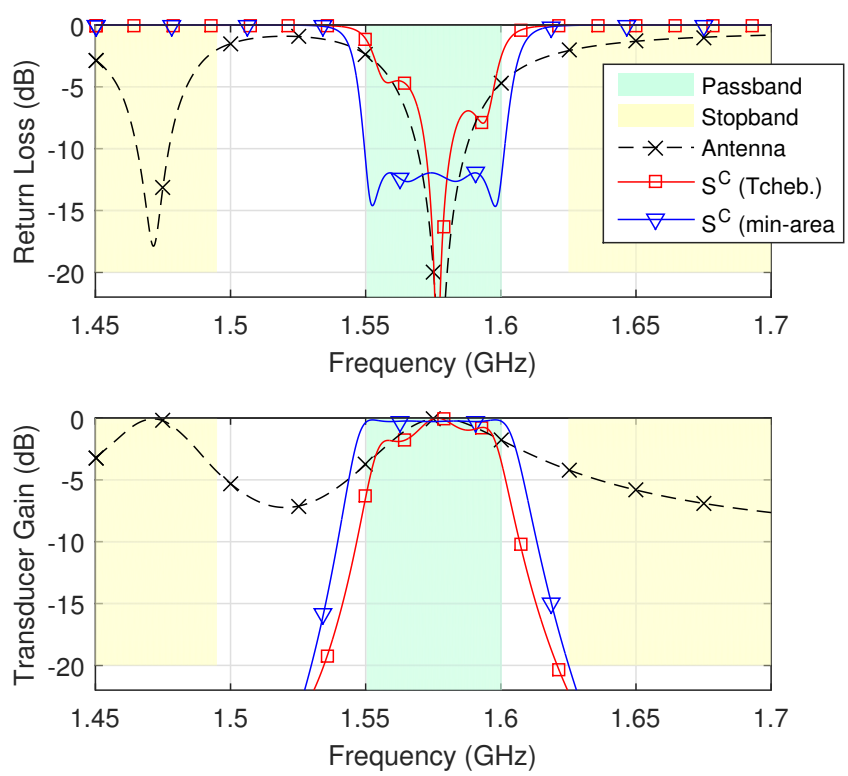

Fig. 3. Reflexion coefficient and transducer gain of the antenna compared to the global system $\left(S^{C}\right)$ in both cases (Tchebyshev and minimum-area).

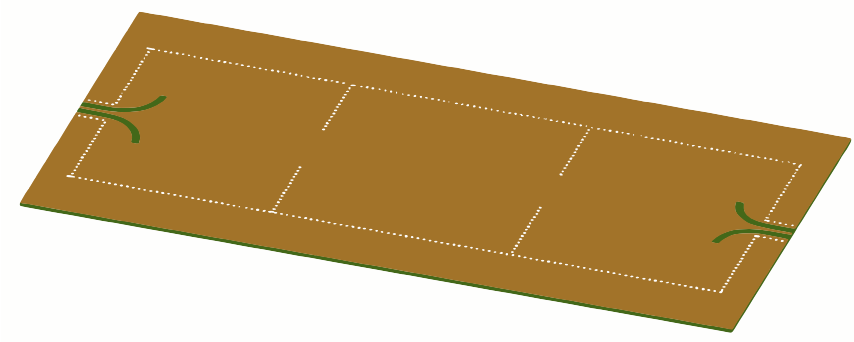

Fig. 4. Structure of the 3-poles substrate-integrated-waveguide (SIW) filter fed with coplanar-waveguide (CPWG) transmission lines designed with the software Ansoft Electronic Desktop and featuring a footprint of 120x40 mm.

Figures 5 and 6 show comparison between the S-parameters of the filter and the overall system, obtained in one case from the EM simulation of the filter and in the other case from its circuital analogue where a quality factor of $Q=200$ has been considered.

The excellent match which is observed validates the employed synthesis and tuning technique for SIW filters and confirms the benefits of using a matching filter.

\section{CONClusion}

An algorithm for the design of matching filters used in connection with antennas has been presented. Provided the reflection coefficient of the antenna can be approached in the band of interest by a rational function of degree one, the presented approach yields the guaranteed best matching response in a given frequency band while satisfying rejection constraints in a prescribed stop band. In this sense it is the generalization of the classical quasi-elliptic synthesis technique for filters
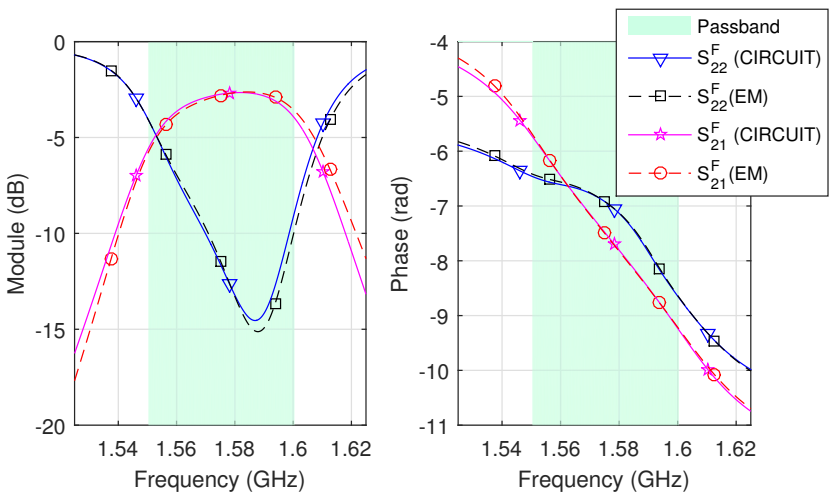

Fig. 5. Result obtained with a SIW filter (circuit and EM)
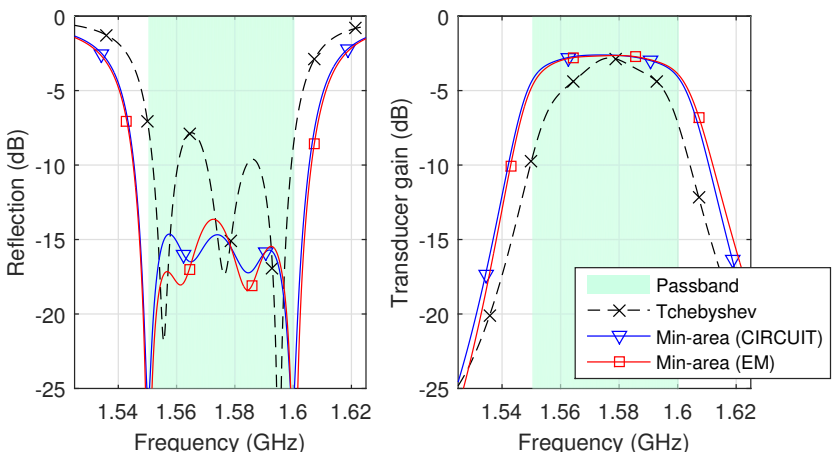

Fig. 6. Matching filter connected to the antenna. (circuit and EM)

matched on a resistive load to the case of filters plugged on a frequency varying load.

To prove the efficiency of this algorithm, a 3-poles matching filter is synthesized to match a microstrip patch antenna. This filter has been designed in SIW technology with excellent results, improving the return loss level of the system from $-7.5 d B$ to $-14 d B$.

\section{ACKNOWLEDGMENT}

This work was supported by the French ANR (National Research Agency).

\section{REFERENCES}

[1] H. Bode, Network Analysis and Feedback Amplifier Design, ser. Bell Telephone Laboratories series. Van Nostrand, 1945.

[2] R. Fano, Theoretical Limitations on the Broadband Matching of Arbitrary Impedances, ser. Technical report: Research Laboratory of Electronics. MIT Res. Lab. of Electronics, 1947.

[3] D. Youla and Polytechnic Institute of Brooklyn NY, A New Theory of Broadband Matching. Defense Technical Information Center, 1964

[4] R. Gudipati and W. K. Chen, "Explicit formulas for the design of broadband matching bandpass equalizers with chebyshev response," in Circuits and Systems, 1995. ISCAS '95., 1995 IEEE International Symposium on, vol. 3, Apr 1995, pp. 1644-1647 vol.3.

[5] H. J. Carlin and P. P. Civalleri, Wideband circuit design, ser. Electronic engineering systems series. Boca Raton, Fla. CRC Press, 1998.

[6] R. Cameron, R. Mansour, and C. Kudsia, Microwave Filters for Communication Systems: Fundamentals, Design and Applications. Wiley, 2007.

[7] Software Presto-HF. Available: https://project.inria.fr/presto-hf/. 